\title{
Refining trigonometric inequalities by using Padé approximant
}

\author{
Zhen Zhang ${ }^{1,2}$, Huaqing Shan ${ }^{2 *}$ and Ligeng Chen ${ }^{2}$
}

"Correspondence:

522947956@qq.com

${ }^{2}$ Key Laboratory of Complex Systems Modeling and Simulation, Hangzhou Dianzi University, Hangzhou, China

Full list of author information is available at the end of the article

\begin{abstract}
A two-point Padé approximant method is presented for refining some remarkable trigonometric inequalities including the Jordan inequality, Kober inequality, Becker-Stark inequality, and Wu-Srivastava inequality. Simple proofs are provided. It shows to achieve better approximation results than those of prevailing methods.
\end{abstract}

Keywords: Trigonometric inequalities; Padé approximant; Two-sided bounds; Rational refinement

\section{Introduction}

Trigonometric inequalities have caused interest of a lot of researchers, they analyzed the Wilker inequality [6-11, 14, 16-19], Jordan inequality [3, 5, 15, 20, 21], Shafer-Fink inequality [12], Becker-Stark inequalities [13], and so on.

Recently, Bercu provided a Padé-approximant-based method and obtained the following inequalities [2].

$$
\begin{aligned}
& b_{1}(x)=\frac{-7 x^{2}+60}{3 x^{2}+60}<\frac{\sin (x)}{x}<\frac{11 x^{4}-360 x^{2}+2520}{60 x^{2}+2520}=b_{2}(x), \quad \forall x \in(0, \pi / 2) \text {; } \\
& b_{3}(x)=\frac{17 x^{4}-480 x^{2}+1080}{2 x^{4}+60 x^{2}+1080}<\cos (x)<\frac{3 x^{4}-56 x^{2}+120}{4 x^{2}+120} \\
& =b_{4}(x), \quad \forall x \in(0, \pi / 2) \text {; } \\
& b_{5}(x)<\frac{\tan (x)}{x}<b_{6}(x), \quad \forall x \in(0,1.5701) ; \\
& \left(\frac{x}{\sin (x)}\right)^{2}+\frac{x}{\tan (x)}>b_{7}(x), \quad \forall x \in(0,1.5701),
\end{aligned}
$$

where $b_{5}(x)=\frac{-28 x^{4}-600 x^{2}+7200}{9 x^{6}+12 x^{4}-3000 x^{2}+7200}, \quad b_{6}(x)=\frac{22 x^{8}-60 x^{6}-4680 x^{4}-237,600 x^{2}+2,721,600}{1020 x^{6}+14,040 x^{4}-1,144,800 x^{2}+2,721,600}$ and $b_{7}(x)=$ $\frac{11,220 x^{10}-205,560 x^{8}-14,256,000 x^{6}+512,179,200 x^{4}-3,157,056,000 x^{2}+13,716,864,000}{242 x^{12}-8580 x^{10}+25,560 x^{8}-1,080,000 x^{6}+103,680,000 x^{4}-1,578,528,000 x^{2}+6,858,432,000}$.

In this paper, we present a two-point Padé-approximant-based method [1] for refining the rational bounds of several trigonometric inequalities, and also provide a method for proving the refined bounds. By applying the new method to $\frac{\sin (x)}{x}$ and $\cos (x)$, we refine the bounds of Eq. (1) (2), for $\forall x \in[0, \pi / 2]$, see also Theorems 3.1 and 3.2. Applied to $\frac{\tan (x)}{x}$ and $\left(\frac{x}{\sin (x)}\right)^{2}+\frac{x}{\tan (x)}$, it not only provides refined two-sided bounds with better approxima-

(c) The Author(s) 2018. This article is distributed under the terms of the Creative Commons Attribution 4.0 International License (http://creativecommons.org/licenses/by/4.0/), which permits unrestricted use, distribution, and reproduction in any medium, provided you give appropriate credit to the original author(s) and the source, provide a link to the Creative Commons license, and indicate if changes were made. 
tion effect for Eq. (3) (4), but also extends the interval $(0,1.5701)$ to the interval $[0, \pi / 2]$, see also the theorems and remarks in Sect. 3.

\section{Find bounds by using two-point Padé approximant}

Given a bounded smooth function $f(x), x \in\left[x_{0}, x_{1}\right]$, let $R(x)=\frac{\sum_{i=0}^{n} c_{i} x^{i}}{1+\sum_{i=1}^{m} d_{i} x^{i}}$ be a rational polynomial interpolating derivatives of $f(x)$ at two points $x_{0}$ and $x_{1}$ such that

$$
E^{(i)}\left(x_{0}\right)=0, \quad E^{(j)}\left(x_{1}\right)=0, \quad i=0,1, \ldots, k, j=0,1, \ldots, l,
$$

where $E(x)=\left(1+\sum_{i=1}^{m} d_{i} x^{i}\right) \cdot f(x)-\left(\sum_{i=1}^{n} c_{i} x^{i}\right)$. There are $m+n+2$ unknowns in Eq. (5). By selecting suitable values of $k$ and $l$, we have that Eq. (5) consists of $m+n+2$ linear equations in the unknown variables $c_{i}$ and $d_{j}$, and the interpolation polynomial $R(x)$ can be determined by solving Eq. (5).

We give two examples. Without loss of generality, let $\Gamma=[0, \pi / 2]$.

Example 1 Let $f_{1}(x)=\sin (x)$. By setting $n_{1}=13, m_{1}=0, n_{2}=11$, and $m_{2}=0$ and introducing the following constraints

$$
f_{1}^{(i)}(0)=R_{j}^{(i)}(0), \quad f_{1}(\pi / 2)=R_{j}(\pi / 2), \quad j=1,2, i=0,1, \ldots, 14-2 j
$$

we obtain that

$$
R_{1}(x)=\beta_{1}(x)+\alpha_{1} \cdot x^{13}, \quad R_{2}(x)=\beta_{2}(x)-\alpha_{2} \cdot x^{11},
$$

where $\alpha_{1}=\frac{\pi^{11}-440 \pi^{9}+126,720 \pi^{7}-21,288,960 \pi^{5}+1,703,116,800 \pi^{3}-40,874,803,200 \pi+81,749,606,400}{9,979,200 \pi^{13}}, \beta_{1}(x)=$ $t-\frac{t^{3}}{6}+\frac{t^{5}}{120}-\frac{t^{7}}{5040}+\frac{t^{9}}{362,880}-\frac{x^{11}}{39,916,800}, \alpha_{2}=\frac{\pi^{9}-288 \pi^{7}+48,384 \pi^{5}-3,870,720 \pi^{3}+92,897,280 \pi-185,794,560}{90,720 \pi^{11}}$, $\beta_{2}(x)=t-\frac{t^{3}}{6}+\frac{t^{5}}{120}-\frac{t^{7}}{5040}+\frac{t^{9}}{362,880}$. It can be verified that $R_{j}(x) \geq 0, \forall x \in \Gamma, j=1,2$. From Eq. (6), $\forall x \in \Gamma$, there exists $\xi_{j}(x) \in \Gamma$ such that [4]

$$
f_{1}(x)-R_{j}(x)=\frac{f_{1}^{(16-2 j)}\left(\xi_{j}(x)\right)}{(16-2 j) !} \cdot(x-\pi / 2) \cdot x^{15-2 j}, \quad x \in \Gamma, j=1,2 .
$$

Note that $f_{1}^{(14)}(x)=-\sin (x) \leq 0$ and $f_{1}^{(12)}(x)=\sin (x) \geq 0, \forall x \in \Gamma$. Combining with Eq. (8), one obtains that

$$
0 \leq R_{1}(x) \leq \sin (x) \leq R_{2}(x), \quad x \in \Gamma .
$$

Example 2 Let $f_{2}(x)=\cos (x)$. By setting $n_{3}=12, m_{3}=0, n_{4}=10$, and $m_{4}=0$ and introducing the following constraints

$$
f_{2}^{(i)}(0)=R_{j}^{(i)}(0), \quad f_{2}(\pi / 2)=R_{j}(\pi / 2), \quad j=3,4, i=0,1, \ldots, 17-2 j,
$$

we obtain that

$$
R_{3}(x)=\beta_{3}(x)+\alpha_{3} \cdot x^{12}, \quad R_{4}(x)=\beta_{4}(x)-\alpha_{4} \cdot x^{10},
$$


where $\alpha_{3}=\frac{\pi^{10}-360 \pi^{8}+80,640 \pi^{6}-9,676,800 \pi^{4}+464,486,400 \pi^{2}-3,715,891,200}{907,200 \pi^{12}}, \beta_{3}(x)=1-\frac{x^{2}}{2}+\frac{x^{4}}{24}-\frac{x^{6}}{720}+$ $\frac{x^{8}}{40,320}-\frac{x^{10}}{3,628,800}, \alpha_{4}=\frac{10,321,920-1,290,240 \pi^{2}+26,880 \pi^{4}-224 \pi^{6}+\pi^{8}}{10,080 \pi^{10}}, \beta_{4}(x)=1-\frac{x^{2}}{2}+\frac{x^{4}}{24}-\frac{x^{6}}{720}+\frac{x^{8}}{40,320}$. It can be verified that $R_{j}(x) \geq 0, \forall x \in \Gamma, j=3,4$. From Eq. (10), $\forall x \in \Gamma$, there exists $\xi_{j}(x) \in$ $\Gamma, j=3,4$, such that $[4]$

$$
f_{2}(x)-R_{j}(x)=\frac{f_{2}^{(19-2 j)}\left(\xi_{j}(x)\right)}{(19-2 j) !} \cdot(x-\pi / 2) \cdot x^{18-2 j}, \quad x \in \Gamma, j=3,4 .
$$

Note that $f_{2}^{(13)}(x)=-\sin (x) \leq 0$ and $f_{2}^{(11)}(x)=\sin (x) \geq 0, \forall x \in \Gamma$. Combining with Eq. (12), one obtains that

$$
0 \leq R_{3}(x) \leq \cos (x) \leq R_{4}(x), \quad x \in \Gamma
$$

\section{Main results}

The main results are as follows.

Theorem 3.1 For all $\forall x \in \Gamma=[0, \pi / 2]$, we have that

$$
\begin{aligned}
{[b] c_{1}(x) } & =\frac{60,480-9240 x^{2}+364 x^{4}-5 x^{6}}{840\left(72+x^{2}\right)} \leq \frac{\sin (x)}{x} \\
& \leq \frac{\left(166,320-22,260 x^{2}+551 x^{4}\right)}{15\left(11,088+364 x^{2}+5 x^{4}\right)}=c_{2}(x)
\end{aligned}
$$

Proof Eq. (14) is equivalent to

$$
\left\{\begin{array}{l}
\left(60,480-9240 x^{2}+364 x^{4}-5 x^{6}\right) x-840\left(72+x^{2}\right) \sin (x) \leq 0, \\
\left(166,320-22,260 x^{2}+551 x^{4}\right) x-15\left(11,088+364 x^{2}+5 x^{4}\right) \sin (x) \geq 0,
\end{array} \quad \forall x \in \Gamma .\right.
$$

It is well known that $\forall x \in \Gamma$,

$$
\begin{aligned}
\beta_{1}(x) & =t-\frac{t^{3}}{6}+\frac{t^{5}}{120}-\frac{t^{7}}{5040}+\frac{t^{9}}{362,880}-\frac{x^{11}}{39,916,800} \\
& \leq \sin (x) \leq \beta_{1}(x)+\frac{x^{13}}{6,227,020,800} .
\end{aligned}
$$

Combining with Eq. (16), we have that

$$
\begin{aligned}
&\left(60,480-9240 x^{2}+364 x^{4}-5 x^{6}\right) x-840\left(72+x^{2}\right) \sin (x) \\
& \leq\left(60,480-9240 x^{2}+364 x^{4}-5 x^{6}\right) x-840\left(72+x^{2}\right) \beta_{1}(x) \\
&= \frac{x^{11} \cdot\left(-38+x^{2}\right)}{39,916,800} \leq 0, \quad \forall x \in \Gamma, \\
&\left(166,320-22,260 x^{2}+551 x^{4}\right) x-15\left(11,088+364 x^{2}+5 x^{4}\right) \sin (x) \\
& \geq \\
& \quad\left(166,320-22,260 x^{2}+551 x^{4}\right) x \\
& \quad-15\left(11,088+364 x^{2}+5 x^{4}\right)\left(\beta_{1}(x)+\frac{x^{13}}{6,227,020,800}\right)
\end{aligned}
$$




$$
\begin{aligned}
& =\frac{x^{11}}{6,227,020,800}\left(1,661,088-40,104 x^{2}+416 x^{4}-5 x^{6}\right) \\
& \geq \frac{x^{11}}{6,227,020,800}\left(1,661,088-40,104 \cdot 2^{2}-5 \cdot 2^{6}\right) \geq 0, \quad \forall x \in \Gamma,
\end{aligned}
$$

which is just Eq. (15). So we have completed the proof of Eq. (14).

Theorem 3.2 For all $\forall x \in[0, \pi / 2]$, we have that

$$
\begin{aligned}
c_{3}(x) & =\frac{20,160-9720 x^{2}+660 x^{4}-13 x^{6}}{360\left(x^{2}+56\right)} \leq \cos (x) \\
& \leq \frac{15,120-6900 x^{2}+313 x^{4}}{15,120+660 x^{2}+13 x^{4}}=c_{4}(x) .
\end{aligned}
$$

Proof Eq. (17) is equivalent to

$$
\left\{\begin{array}{l}
\left(20,160-9720 x^{2}+660 x^{4}-13 x^{6}\right)-360\left(x^{2}+56\right) \cos (x) \leq 0, \\
\left(15,120-6900 x^{2}+313 x^{4}\right)-\left(15,120+660 x^{2}+13 x^{4}\right) \cos (x) \geq 0,
\end{array} \quad \forall x \in \Gamma .\right.
$$

It is well known that

$$
\begin{aligned}
\beta_{3}(x) & =1-\frac{x^{2}}{2}+\frac{x^{4}}{24}-\frac{x^{6}}{720}+\frac{x^{8}}{40,320}-\frac{x^{10}}{3,628,800} \leq \cos (x) \\
& \leq 1-\frac{x^{2}}{2}+\frac{x^{4}}{24}-\frac{x^{6}}{720}+\frac{x^{8}}{40,320}=\beta_{4}(x), \quad \forall x \in \Gamma .
\end{aligned}
$$

Combining with Eq. (19), we have that

$$
\left\{\begin{array}{l}
\left(20,160-9720 x^{2}+660 x^{4}-13 x^{6}\right)-360\left(x^{2}+56\right) \cos (x) \\
\quad \leq\left(20,160-9720 x^{2}+660 x^{4}-13 x^{6}\right)-360\left(x^{2}+56\right) \beta_{3}(x) \\
\quad=\frac{x^{10}}{3,628,800}\left(-34+x^{2}\right) \leq 0, \quad \forall x \in[0, \pi / 2], \\
\left(15,120-6900 x^{2}+313 x^{4}\right)-\left(15,120+660 x^{2}+13 x^{4}\right) \cos (x) \\
\geq\left(15,120-6900 x^{2}+313 x^{4}\right)-\left(15,120+660 x^{2}+13 x^{4}\right) \beta_{4}(x) \\
=\frac{x^{10}}{40,320}\left(68-13 x^{2}\right) \geq 0, \quad \forall x \in[0, \pi / 2] .
\end{array}\right.
$$

Thus, we have completed the proof of both Eq. (18) and Eq. (17).

Theorem 3.3 For all $\forall x \in \Gamma$, we have that

$$
\begin{aligned}
c_{5}(x) & =\frac{21\left(495-60 x^{2}+x^{4}\right)}{10,395-4725 x^{2}+210 x^{4}-x^{6}} \leq \frac{\tan (x)}{x} \\
& \leq \frac{T_{1}(x)}{105\left(\pi^{2}-4 x^{2}\right) \cdot T_{2}(x)}=c_{6}(x),
\end{aligned}
$$

where $T_{1}(x)=\left(\pi^{6}-840 \pi^{4}+75,600 \pi^{2}-665,280\right) x^{6}+\left(210 \pi^{6}+52,920 \pi^{4}-7,620,480 \pi^{2}+\right.$ $69,854,400) x^{4}+\left(-17,955 \pi^{6}+1,323,000 \pi^{4}+52,390,800 \pi^{2}-628,689,600\right) x^{2}+\left(155,925\left(\pi^{4}-\right.\right.$ $\left.\left.112 \pi^{2}+1008\right)\right) \pi^{2}$ and $T_{2}(x)=\left(26 \pi^{4}-2664 \pi^{2}+23,760\right) x^{4}+\left(-666 \pi^{4}+73,980 \pi^{2}-\right.$ $665,280) x^{2}+\left(1485 \pi^{4}-166,320 \pi^{2}+1,496,880\right)$. 
Proof Eq. (21) is equivalent to

$$
\left\{\begin{array}{rl}
H_{5}(x)= & 21\left(495-60 x^{2}+x^{4}\right) \cdot x \cos (x) \\
& -\left(10,395-4725 x^{2}+210 x^{4}-x^{6}\right) \cdot \sin (x) \leq 0 ; \\
H_{6}(x)= & 105\left(\pi^{2}-4 x^{2}\right) \cdot T_{2}(x) \cdot \sin (x)-T_{1}(x) \cdot x \cos (x) \leq 0,
\end{array} \quad \forall x \in \Gamma .\right.
$$

It can be verified that

$$
\left\{\begin{array}{l}
\cos (x) \leq 1-\frac{x^{2}}{2}+\frac{x^{4}}{24}-\frac{x^{6}}{720}+\frac{x^{8}}{40,320}-\frac{x^{10}}{3,628,800}+\frac{x^{12}}{479,001,600}=\beta_{5}(x), \\
\beta_{1}(x)=t-\frac{t^{3}}{6}+\frac{t^{5}}{120}-\frac{t^{7}}{5040}+\frac{t^{9}}{362,880}-\frac{x^{11}}{39,916,800} \leq \sin (x), \\
495-60 x^{2}+x^{4}>0, \quad 10,395-4725 x^{2}+210 x^{4}-x^{6}>0,
\end{array} \quad \forall x \in \Gamma .\right.
$$

Combining with Eq. (23), we have that

$$
\begin{aligned}
H_{5}(x) & \leq 21\left(495-60 x^{2}+x^{4}\right) \cdot x \beta_{5}(x)-\left(10,395-4725 x^{2}+210 x^{4}-x^{6}\right) \cdot \beta_{1}(x) \\
& =\frac{x^{13}}{159,667,200}\left(-915-64 x^{2}+3 x^{4}\right) \leq 0, \quad \forall x \in \Gamma
\end{aligned}
$$

Let $\beta_{6}(x)=T_{1}(x)+105\left(\pi^{2}-4 x^{2}\right) \cdot T_{2}^{\prime}(x)-840 x \cdot T_{2}(x), \beta_{7}(x)=105\left(\pi^{2}-4 x^{2}\right) \cdot T_{2}(x)-T_{1}^{\prime}(x)$.

On the other hand, it can be verified that, $\forall x \in \Gamma$,

$$
\begin{aligned}
& H_{6}^{\prime}(x)=\beta_{6}(x) \cdot \sin (x)+\beta_{7}(x) \cdot \cos (x), \\
& \beta_{6}(x) \leq 0, \quad \beta_{7}(x) \geq 0, \quad T_{2}(x) \geq 0, \quad T_{1}(x) \geq 0, \\
& \cos (x) \geq 1-\frac{x^{2}}{2}+\frac{x^{4}}{24}-\frac{x^{6}}{720}+\frac{x^{8}}{40,320}-\frac{x^{10}}{3,628,800}+\frac{x^{12}}{479,001,600} \\
& \quad-\frac{x^{14}}{87,178,291,200}=\beta_{8}(x), \\
& \beta_{9}(x)=t-\frac{t^{3}}{6}+\frac{t^{5}}{120}-\frac{t^{7}}{5040}+\frac{t^{9}}{362,880}-\frac{x^{11}}{39,916,800}+\frac{x^{13}}{6,227,020,800} \geq \sin (x) .
\end{aligned}
$$

Combining Eq. (23) with Eq. (25), we have that

$$
\begin{aligned}
H_{6}(x) & \leq 105\left(\pi^{2}-4 x^{2}\right) \cdot T_{2}(x) \cdot \beta_{9}(x)-T_{1}(x) \cdot x \beta_{8}(x) \\
& =\frac{x^{13}}{9,153,720,576,000} \beta_{10}(x) \leq 0, \quad \forall x \in\left[0, \frac{31 \pi}{64}\right], \\
H_{6}^{\prime}(x) & \geq \beta_{6}(x) \cdot \beta_{1}(x)+\beta_{7}(x) \cdot \beta_{5}(x) \\
& =\frac{x^{12}}{50,295,168,000} \beta_{11}(x) \geq 0, \quad \forall x \in\left[\frac{31 \pi}{64}, \frac{\pi}{2}\right],
\end{aligned}
$$

where $\beta_{10}(x)=\left(18,063,360 \pi^{6}-8,128,512,000 \pi^{4}+643,778,150,400 \pi^{2}-5,579,410,636,800\right)+$ $\left(-634,725 \pi^{6}+305,912,880 \pi^{4}-24,700,198,320 \pi^{2}+214,592,716,800\right) x^{2}+\left(6069 \pi^{6}-4,639,320 \pi^{4}+\right.$ $\left.411,823,440 \pi^{2}-3,618,457,920\right) x^{4}+\left(28 \pi^{6}+52,920 \pi^{4}-5,715,360 \pi^{2}+51,226,560\right) x^{6}+\left(\pi^{6}-\right.$ $\left.840 \pi^{4}+75,600 \pi^{2}-665,280\right) x^{8} \leq 0, \forall x \in\left[0, \frac{31 \pi}{64}\right], \beta_{11}(x)=\left(-1,290,240 \pi^{6}+580,608,000 \pi^{4}-\right.$ $\left.45,984,153,600 \pi^{2}+398,529,331,200\right)+\left(54,405 \pi^{6}-25,552,800 \pi^{4}+2,048,684,400 \pi^{2}-\right.$ 
$17,782,934,400) x^{2}+\left(-1404 \pi^{6}+556,920 \pi^{4}-42,366,240 \pi^{2}+365,238,720\right) x^{4}+\left(19 \pi^{6}-5040 \pi^{4}+\right.$ $\left.317,520 \pi^{2}-2,661,120\right) x^{6} \geq 0, \forall x \in\left[\frac{31 \pi}{64}, \frac{\pi}{2}\right]$. Combining Eq. (26) with $H_{6}(\pi / 2)=0$, we obtain that

$$
H_{6}(x) \leq 0, \quad \forall x \in\left[0, \frac{\pi}{2}\right]
$$

Combining Eq. (24) with Eq. (27), we have completed the proof of both Eq. (22) and Eq. (21).

From Theorems 3.1, 3.2, and 3.3, we directly obtain the following theorem.

Theorem 3.4 We have that

$$
\frac{1}{c_{2}(x)^{2}}+\frac{1}{c_{6}(x)} \leq\left(\frac{x}{\sin (x)}\right)^{2}+\frac{x}{\tan (x)} \leq \frac{1}{c_{1}(x)^{2}}+\frac{1}{c_{5}(x)}, \quad \forall x \in[0, \pi / 2]
$$

\section{Discussion and conclusions}

Firstly, we compare the results of $\frac{\sin (x)}{x}$ between $b_{i}(x)$ in [2] and $c_{i}(x)$ in this paper, $i=1,2$. It can be verified that $c_{1}(x)-b_{1}(x)=\frac{x^{6}\left(264-5 x^{2}\right)}{840\left(72+x^{2}\right)\left(x^{2}+20\right)} \geq 0$ and $c_{2}(x)-b_{2}(x)=$ $\frac{-11 x^{8}}{12\left(11,088+364 x^{2}+5 x^{4}\right)\left(x^{2}+42\right)} \leq 0, \forall x \in[0, \pi / 2]$, we have that

$$
b_{1}(x) \leq c_{1}(x) \leq \frac{\sin (x)}{x} \leq c_{2}(x) \leq b_{2}(x), \quad \forall x \in[0, \pi / 2] .
$$

Secondly, we compare the approximation results of $\cos (x)$ between previous $b_{i}(x)$ and present $c_{i}(x), i=3$, 4 . It can be verified that $c_{3}(x)-b_{3}(x)=\frac{x^{8}\left(270-13 x^{2}\right)}{360\left(56+x^{2}\right)\left(x^{4}+30 x^{2}+540\right)} \geq 0$ and $c_{2}(x)-b_{2}(x)=\frac{-39 x^{8}}{4\left(15,120+660 x^{2}+13 x^{4}\right)\left(x^{2}+30\right)} \leq 0, \forall x \in[0, \pi / 2]$, we have that

$$
b_{3}(x) \leq c_{3}(x) \leq \cos (x) \leq c_{4}(x) \leq b_{4}(x), \quad \forall x \in[0, \pi / 2]
$$

Thirdly, we compare the approximation results of $\frac{\tan (x)}{x}$, which also shows that this paper achieves a much better result. It can be verified that $\forall x \in[0, \pi / 2]$,

$$
c_{5}(x)-b_{5}(x)=\frac{x^{6}\left(161 x^{2}-495\right)\left(x^{2}-33\right)}{3\left(10,395-4725 x^{2}+210 x^{4}-x^{6}\right)\left(x^{2}+20\right)\left(3 x^{4}-56 x^{2}+120\right)} \geq 0 .
$$

However, note that the denominator of $b_{6}(x)$ is $T_{3}(x)=1020 x^{6}+14,040 x^{4}-1,144,800 x^{2}+$ $2,721,600=30\left(17 x^{4}-480 x^{2}+1080\right)\left(x^{2}+42\right)$, which has a real root $\approx 1.5701$ within the interval $\Gamma$, and we have $T_{3}(x)>0, \forall x \in[0,1.5701]$. It can be verified that $c_{6}(x)-$ $b_{6}(x)=\frac{-x^{8} H_{7}(x)}{210 T_{2}(x) T_{3}(x)\left(\pi^{2}-4 x^{2}\right)}$, where $H_{7}(x)=378,675\left(\pi^{4}-112 \pi^{2}+1008\right) \pi^{2}+\left(-64,350 \pi^{6}+\right.$ $\left.5,536,440 \pi^{4}+106,323,840 \pi^{2}-1,526,817,600\right) x^{2}+\left(1968 \pi^{6}+50,400 \pi^{4}-25,764,480 \pi^{2}+\right.$ $247,484,160) x^{4}+\left(-8008 \pi^{4}+820,512 \pi^{2}-7,318,080\right) x^{6}$. By using the Maple software, $H_{7}(x)$ has six real roots $\approx-9.16,-4.97,-2.76,2.76,4.97,9.16$, and $H_{7}(x), T_{2}(x), T_{3}(x)>0, \forall x \in$ $(0,1.5701)$, we have that

$$
c_{6}(x)-b_{6}(x) \leq 0, \quad \forall x \in[0,1.5701]
$$


Competing interests

The authors declare that they have no competing interests.

\section{Authors' contributions}

All authors contributed equally to the manuscript and read and approved the final manuscript.

\section{Author details}

'School of Cyberspace, Hangzhou Dianzi University, Hangzhou, China. ${ }^{2}$ Key Laboratory of Complex Systems Modeling and Simulation, Hangzhou Dianzi University, Hangzhou, China.

\section{Publisher's Note}

Springer Nature remains neutral with regard to jurisdictional claims in published maps and institutional affiliations.

Received: 16 March 2018 Accepted: 22 May 2018 Published online: 27 June 2018

\section{References}

1. Baker, G.A. Jr., Graves-Morris, P.: Padé Approximants. Cambridge University Press, New York (1996)

2. Bercu, G.: The natural approach of trigonometric inequalities-Padé approximant. J. Math. Inequal. 11(1), 181-191 (2017)

3. Chen, C.P., Debnath, L.: Sharpness and generalization of Jordan's inequality and its application. Appl. Math. Lett. 25(3), 594-599 (2012)

4. Davis, P.J.: Interpolation and Approximation. Dover Publications, New York (1975)

5. Debnath, L., Mortici, C., Zhu, L.: Refinements of Jordan-Steckin and Becker-Stark inequalities. Results Math. 67(1-2), 207-215 (2015)

6. Jiang, W.D., Luo, Q.M., Qi, F.: Refinements and sharpening of some Huygens and Wilker type inequalities. Math. Inequal. Appl. 6(1), 19-22 (2014)

7. Lutovac, T., Malešsević, B., Mortici, C.: The natural algorithmic approach of mixed trigonometric-polynomial problems. J. Inequal. Appl. 2017, 116 (2017)

8. Malešević, B., Makragic, M.: A method for proving some inequalities on mixed trigonometric polynomial functions. J. Math. Inequal. 10, 849-876 (2015)

9. Mortici, C.: The natural approach of Wilker-Cusa-Huygens inequalities. Math. Inequal. Appl. 14, 535-541 (2011)

10. Mortici, C.: A subtly analysis of Wilker inequality. Appl. Math. Comput. 231, 516-520 (2014)

11. Nenezić, M., Malesević, B., Mortici, C.: New approximations of some expressions involving trigonometric functions. Appl. Math. Comput. 283, 299-315 (2016)

12. Nishizawa, Y.: Sharpening of Jordan's type and Shafer-Fink's type inequalities with exponential approximations. Appl. Math. Comput. 269, 146-154 (2015)

13. Sun, Z.J., Zhu, L.: Simple proofs of the Cusa-Huygens-type and Becker-Stark-type inequalities. J. Math. Inequal. 7(4), 563-567 (2013)

14. Wilker, N.E.: Huygens-type inequalities for the generalized trigonometric and for the generalized hyperbolic functions. Appl. Math. Comput. 230(3), 211-217 (2014)

15. Wu, S., Debnath, L.: A new generalized and sharp version of Jordan's inequality and its applications to the improvement of the Yang Le inequality. Appl. Math. Lett. 20(5), 532-538 (2007)

16. Wu, S.-H., Srivastava, H.M.: A weighted and exponential generalization of Wilker's inequality and its applications. Integral Transforms Spec. Funct. 18(7-8), 529-535 (2007)

17. Wu, S.H., Li, S.G., Bencze, M.: Sharpened versions of Mitrinovic-Adamovic, Lazarevic and Wilker's inequalities for trigonometric and hyperbolic functions. J. Nonlinear Sci. Appl. 9(5), 2688-2696 (2016)

18. Wu, S.H., Yu, H.P., Deng, Y.P., et al.: Several improvements of Mitrinovic-Adamovic and Lazarevic's inequalities with applications to the sharpening of Wilker-type inequalities. J. Nonlinear Sci. Appl. 9(4), 1755-1765 (2016)

19. Yang, Z.H., Chu, Y.M., Zhang, X.H.: Sharp Cusa type inequalities with two parameters and their applications. Appl. Math. Comput. 268, 1177-1198 (2015)

20. Zhu, L.: Sharpening Jordan's inequality and the Yang Le's inequality. Appl. Math. Lett. 19(3), 240-243 (2006)

21. Zhu, L.: Sharpening Jordan's inequality and Yang Le's inequality. II. Appl. Math. Lett. 19(9), 990-994 (2006)

\section{Submit your manuscript to a SpringerOpen ${ }^{\odot}$ journal and benefit from:}

- Convenient online submission

- Rigorous peer review

- Open access: articles freely available online

- High visibility within the field

- Retaining the copyright to your article

Submit your next manuscript at $\boldsymbol{~ s p r i n g e r o p e n . c o m ~}$ 